\title{
A NONUNITARY PAIRING OF POLARIZATIONS FOR THE KEPLER PROBLEM
}

\author{
BY
}

\section{J. H. RAWNSLEY}

\begin{abstract}
The half-form pairing of two polarizations of the Kepler manifold is found and shown to define a bounded linear isomorphism of the two Hilbert spaces, but is not unitary.
\end{abstract}

1. Introduction. In [15] J.-M. Souriau showed that, when suitably completed, the phase space and flow of the Kepler problem in $n$ dimensions could be identified with $T_{0}^{*} S^{n}$ (the cotangent bundle of the $n$-sphere minus its zero section), and its geodesic flow (for the standard metric). This extended a similar result of $\mathbf{J}$. Moser [7] concerning the energy surfaces. Souriau also observed that $T_{0}^{*} S^{n}$ had a complex structure invariant under the flow of the length function. In [10] I showed that this complex structure was a positive polarization for the natural symplectic structure of the cotangent bundle and therefore determines a quantization of the flow [6], [13], [14].

$T_{0}^{*} S^{n}$ has a real polarization, given by the cotangent fibres, but this is not invariant under the flow. By using the method of moving polarizations, $J$. Elhadad [3] quantized a related flow, but for the flow I consider there is an obstruction to the formal pairing noticed by R. Blattner [2]. There is no obstruction to the pairing of the real and complex polarizations, so we can use the transformation defined by the pairing [2], [5], [6] to carry the quantization of the flow from the complex to the real polarization. The generator of the unitary group so obtained on $L^{2}\left(S^{n}\right)$ is $2 \pi[-\Delta+(n-$ $\left.1)^{2} / 4\right]^{1 / 2}$ which has spectrum $2 \pi(k+(n-1) / 2), k=0,1,2, \ldots$ This agrees with the semiclassical spectrum of A. Weinstein [16] but has different multiplicities.

The pairing of these two polarizations is of interest since it is not unitary. It requires some tedious computations to establish it as a bounded linear operator between the Hilbert spaces of the two polarizations. It is closely related to the Laplace representation of spherical harmonics [8].

This paper is divided up as follows: $\$ 2$ summarizes the theory of polarizations and half-form pairings and as an example I obtain Bargmann's transform [1] between the real and complex polarizations of $\mathbf{R}^{2 n} \cong \mathbf{C}^{n}$. The real

Received by the editors April 4, 1977 and, in revised form, January 25, 1978.

AMS (MOS) subject classifications (1970). Primary 53C15, 58A30; Secondary 33A45. 
and complex polarizations of $T_{0}^{*} S^{n}$ together with the formal expression for their pairing are described in $\$ 3$. The rigorous existence and nonunitary nature of the pairing is established in $\$ 4$. An appendix contains the evaluation of some integrals required in $\$ 4$.

I thank the referee for suggestions which have considerably improved the presentation of the original manuscript. Thanks are also due to $\mathbf{R}$. Blattner and D. Simms for their help and interest in this work.

2. Polarizations and the half-form pairing. If $(X, \omega)$ is a symplectic manifold, the space $C(X)$ of complex functions on $X$ is a Lie algebra under Poisson bracket:

$$
\left.[\varphi, \psi]=\xi_{\varphi} \psi ; \quad \xi_{\varphi}\right\lrcorner \omega=d \varphi .
$$

If $\omega$ determines an integral de Rham cohomology class, there is a Hermitian line bundle $L$ with connection $\nabla$ over $X$ having curvature $2 \pi i \omega$. The space $\Gamma L$ of sections of $L$ is a $C(X)$-module where, for $\varphi \in C(X), s \in \Gamma L$

$$
\varphi \cdot s=\nabla_{\xi_{\phi}} s+2 \pi i \varphi s .
$$

This representation of $C(X)$ is known as prequantization. See [4] for details.

A polarization of $(X, \omega)$ is a subbundle $F$ of the complexified tangent bundle $T X^{\mathrm{C}}$ which is

(i) isotropic;

(ii) maximal with respect to (i);

(iii) integrable.

Condition (i) means $\omega$ vanishes identically when restricted to $F$. If $\operatorname{dim} X=$ $2 n$, then by (ii) $\operatorname{dim} F_{x}=n$ for all $x \in X$. If $F^{0} \subset T^{*} X^{\mathbf{C}}$ denotes the bundle of covectors vanishing on $F$, then (i) and (ii) are equivalent to $\xi \mapsto \xi\lrcorner \omega$ maps $F$ isomorphically onto $F^{0}$. We shall take integrable to mean: $F \cap \bar{F}$ has constant dimension and $F, F+\bar{F}$ are closed under the Lie bracket of vector fields. Thus the complex Frobenius theorem of Nirenberg [9] applies to $F$.

There are two main examples of polarizations. If $F=\bar{F}, F$ is called real and is the tangent bundle of a Lagrangian foliation of $(X, \omega)$. The fibres of a cotangent bundle $X=T^{*} M$ is a typical example of this situation. At the other extreme we may have $F \cap \bar{F}=0$, in which case $T X^{\mathrm{C}}=F \oplus \bar{F}$ so that an almost complex structure $J$ may be defined on $X$ in such a way that $F$ consists of tangents of type $(0,1)$. Since $F$ is involutive, $J$ is integrable and $X$ becomes a complex manifold.

$$
g(\xi, \eta)=\omega(J \xi, \eta), \quad \xi, \eta \in \Gamma T X,
$$

defines a nonsingular symmetric bilinear form on the tangent spaces to $X$ which is Hermitian for the complex structure. The associated 2-form is $\omega$ which is closed, so that $g$ is a (pseudo-) Kaehler metric. Thus any Kaehler manifold is an example of a symplectic manifold with a polarization. 
If $F$ is a polarization of $(X, \omega)$ it is called positive if $-i \omega(\xi, \bar{\xi})>0$, $\forall \xi \in \Gamma F$. Real polarizations are always positive, whilst if $F \cap \bar{F}=0, F$ is positive if and only if $g$ is positive definite.

Given a polarization $F$ of $(X, \omega)$ we can define the structure sheaf $\mathcal{C}_{F}$ as the sheaf associated to the presheaf

$$
U \mapsto C_{F}(U)=\{\varphi \in C(U) \mid \xi \varphi=0, \forall \xi \in \Gamma F\}, \quad U \subset X \text { open. }
$$

See [6], [12] for some properties of this sheaf. When $F \cap \bar{F}=0, \mathcal{C}_{F}$ is the sheaf of holomorphic functions on $X$.

Let $L, \nabla$ be a prequantization of $(X, \omega)$ and $F$ a polarization, then we set

$$
\Gamma_{F} L=\left\{s \in \Gamma L \mid \nabla_{\xi} s=0, \forall \xi \in \Gamma F\right\} .
$$

$\Gamma_{F} L$ is not stable under all $\varphi \in C(X)$, but those functions $\varphi$ which preserve $\Gamma_{F} L$ form a Lie subalgebra $C_{F}^{1}(X)$ which contains $C_{F}(X)$ as a maximal abelian ideal. The representation of $C_{F}^{1}(X)$ on $\Gamma_{F} L$ is called the quantization with respect to $F$.

If $U \subset X$ is open with $H^{1}\left(U, \bigodot_{F}\right)=0$ and $\omega \mid U=d \theta$ with $\theta \mid F=0$ then there is a nowhere vanishing section $s$ of $L$ over $U$ with $\nabla_{\xi} s=2 \pi i \theta(\xi) s$ for all vector fields $\xi . \Gamma_{F}(L \mid U)$ can be identified with $C_{F}(U)$ by $\varphi \mapsto \varphi s, \varphi \in C_{F}(U)$ and if $\psi \in C_{F}^{1}(U)$

$$
\psi(\varphi s)=\left\{[\psi, \varphi]+2 \pi i\left(\theta\left(\xi_{\psi}\right)+\psi\right) \varphi\right\} s .
$$

In general it is difficult to make $\Gamma_{F} L$ into a Hilbert space, which is desirable if this construction is going to be used to construct the quantum mechanical model corresponding with the classical system described by $(X, \omega)$. Even when this is possible there is no way of comparing $\Gamma_{F} L$ with $\Gamma_{G} L$ for different polarizations $F$ and $G$. For these reasons B. Kostant introduced the notation of half-forms and their pairing in [5], [6], and this was further developed by R. Blattner [2]. There is no satisfactory theory at present unless $F$ and $G$ are both positive. The formalism we shall use is that of [11].

If $F$ is a polarization of $(X, \omega), \operatorname{dim} X=2 n$, then $\Lambda^{n} F^{0}$ is a line bundle, the canonical bundle $K^{F}$ of $F$. If $F \cap \bar{F}=0, K^{F}$ is the canonical bundle of the complex structure. For $F$ positive the Chern class of $K^{F}$ is determined by $\omega$ so that $K^{F}$ and $K^{G}$ are isomorphic as $C^{\infty}$ line bundles for any two positive polarizations $F$ and $G$. In this case $K^{F} \otimes \overline{K^{G}}$ is trivial, and a pairing of $K^{F}$ with $K^{G}$ is a choice of a trivialization of this bundle.

When $F \cap \bar{G}=0$ exterior multiplication defines an isomorphism of $K^{F} \otimes \overline{K^{G}}$ with $\Lambda^{2 n} T^{*} X^{\mathrm{C}}$ and the latter is trivialized by the Liouville volume $\lambda=(-1)^{n(n-1) / 2} \omega^{n} / n !$. Hence if $\alpha \in \Gamma K^{F}, \beta \in \Gamma K^{G}$ we define $\langle\alpha, \beta\rangle$ by

$$
i^{n}\langle\alpha, \beta\rangle \lambda=\alpha \wedge \bar{\beta} .
$$

If $F \cap \bar{G}$ has constant rank then $F \cap \bar{G}=D^{\mathbf{C}}$ for a real integrable isotropic subbundle $D$ of $T X$ (positivity of $F$ and $G$ is required here). Let $D^{\perp}$ 
denote all $\xi \in T X$ with $\omega(\xi, D)=0$, then $D \subset D^{\perp}$ and $\omega$ induces a nonsingular skew form $\omega / D$ on $D^{\perp} / D$ making $D^{\perp} / D$ a symplectic vector bundle. Since $D \subset F, F \subset\left(D^{\perp}\right)^{\mathrm{C}}$ so projects to give a maximal isotropic subbundle $F / D$ of $\left(D^{\perp} / D\right)^{\mathrm{C}}$. The same is true of $G$, and $F / D \cap \overline{G / D}=0$. Then $K^{F / D}$ and $K^{G / D}$ are paired by exterior multiplication as above. We lift this pairing to $K^{F}$ and $K^{G}$ as follows:

Let $b=\left(e_{1}, \ldots, e_{k}\right)$ be a frame for $D_{x}$. Then it can be extended to a frame $\left(e_{1}, \ldots, e_{k}, f_{1}, \ldots, f_{n-k}\right)$ for $F_{x}$ and if $\alpha \in K_{x}^{F}$,

$$
\left.\left.\left.\left.\alpha=a\left(e_{1}\right\lrcorner \omega\right) \wedge \cdots \wedge\left(e_{k}\right\lrcorner \omega\right) \wedge\left(f_{1}\right\lrcorner \omega\right) \wedge \cdots \wedge\left(f_{n-k}\right\lrcorner \omega\right)
$$

for some $a \in \mathrm{C}$. Let $\tilde{f}_{i}$ be the projection of $f_{i} \in\left(D_{x}^{\perp}\right)^{\mathbf{C}}$ into $\left(D^{\perp} / D\right)_{x}^{\mathbf{C}}$ so that $\left(\tilde{f}_{1}, \ldots, \tilde{f}_{n-k}\right)$ is a frame for $(F / D)_{x}$. Put

$$
\left.\left.\tilde{\alpha}_{b}=a\left(\tilde{f}_{1}\right\lrcorner \omega / D\right) \wedge \cdots \wedge\left(\tilde{f}_{n-k}\right\lrcorner \omega / D\right) \in K_{x}^{F / D} .
$$

Then $\tilde{\alpha}_{b}$ does not depend on the extension $f_{1}, \ldots, f_{n-k}$ and if $g \in \mathrm{GL}(k, \mathbf{R})$, $\tilde{\alpha}_{b \cdot g}=\operatorname{Det}\left[g^{-1}\right] \tilde{\alpha}_{b}$. We can project $\beta \in K_{x}^{G}$ in the same fashion. Put $\langle\alpha, \beta\rangle(b)=\left\langle\tilde{\alpha}_{b}, \tilde{\beta}_{b}\right\rangle$. Then $\langle\alpha, \beta\rangle$ is a density of order -2 on $D$ and, using the Liouville density on $T X$, defines a density of order 2 on $(T X) / D$.

Let us suppose the space $X / D$ of leaves of the foliation $D$ is smooth then $(T X) / D$ is the pull back to $X$ of the tangent bundle $T(X / D)$. If $\langle\alpha, \beta\rangle$ is covariant constant along the leaves it will project down to a density of order 2 on $X / D$. If we could everywhere take a square root we should end with a density of order 1 on $X / D$ which would be a candidate for integrating over $X / D$ to obtain a global pairing.

There are clearly many points at which this procedure can break down. First, $K^{F}$ may not have a square root. It has one precisely when its Chern class is divisible by 2 (in which case $(X, \omega)$ is called metaplectic). Assuming this is so, the symplectic frame bundle of $(X, \omega)$ has a double covering from which a square root $Q^{F}$ of $K^{F}$ can be canonically constructed for each positive polarization $F$. These square roots have the property that $Q^{F} \otimes \overline{Q^{G}}$ is trivial, which is necessary if a pairing is to exist. See [2] for the construction. Sections of $Q^{F}$ are called half-forms normal to $F$.

There is a pairing $\langle\cdot, \cdot\rangle$ of $Q^{F} \otimes \overline{Q^{G}}$ into the densities of order -1 on $D$ such that for $\mu \in \Gamma Q^{F}, \nu \in \Gamma Q^{G},\langle\mu, \nu\rangle^{2}=\langle\mu \otimes \mu, \nu \otimes \nu\rangle$.

The procedure now is to replace $L$ by $L \otimes Q^{F}$, and define $\Gamma_{F} L \otimes Q^{F}$ by introducing a covariant derivative in $Q^{F}$. It is fortunate that $Q^{F}$ has a covariant derivative along $F$ arising from Lie differentiation in $K^{F}$. If $\xi \in \Gamma F$, $\alpha \in \Gamma K^{F}$ then $\left.\nabla_{\xi} \alpha=\xi\right\lrcorner d \alpha$ defines $\nabla_{\xi}$ in $\Gamma K^{F}$ and

$$
\nabla_{\xi}\left(\mu_{1} \otimes \mu_{2}\right)=\left(\nabla_{\xi}^{1 / 2} \mu_{1}\right) \otimes \mu_{2}+\left(\mu_{1} \otimes \nabla_{\xi}^{1 / 2} \mu_{2}\right)
$$

defines $\nabla_{\xi}^{1 / 2}$ uniquely in $\Gamma Q^{F}$. Then $\nabla \otimes 1+1 \otimes \nabla^{1 / 2}$ defines a connection along $F$ in $L \otimes Q^{F}$ and $\Gamma_{F} L \otimes Q^{F}$ is defined as before. 
$\Gamma_{F} L \otimes Q^{F}$ is paired with $\Gamma_{G} L \otimes Q^{G}$ by pairing $L$ with itself using the Hermitian structure and $Q^{F}$ with $Q^{G}$ using $\langle\cdot, \cdot\rangle$. Lie differentiation defines a connection along $D$ in the densities on $(T X) / D$, but Blattner found that, in general, $\nabla_{\xi}\langle\rho, \sigma\rangle$ need not vanish for $\rho \in \Gamma_{F} L \otimes Q^{F}, \sigma \in \Gamma_{G} L \otimes Q^{G}$. In all the cases we are interested in $\langle\rho, \sigma\rangle$ does project to a density on $X / D$ so we shall not investigate this point further.

To obtain the inner product in $\Gamma_{F} L \otimes Q^{F}$ one pairs $F$ to itself. Let $\mathfrak{G}_{F}$ be the resulting Hilbert space (which may consist only of zero). If $F \cap \bar{F}=D^{\mathbf{C}}$ the inner product involves integrating over $X / D$. If $F \cap \bar{F}=0$ this is integration over $X$.

As an example take $X=\mathbf{R}^{2 n}, \omega=\sum_{i=1}^{n} d p_{i} \wedge d q_{i}$ where we take $\left(q_{1}, \ldots, q_{n}, p_{1}, \ldots, p_{n}\right)$ as coordinates. Then $F$, spanned by $\partial / \partial p_{1}, \ldots, \partial / \partial p_{n}$, is a real polarization and $K^{F}$ is spanned by $d q_{1}$ $\wedge \cdots \wedge d q_{n}$ so is trivial. Let $Q^{F}$ be spanned by $\left(d q_{1} \wedge \cdots \wedge d q_{n}\right)^{1 / 2}$ (defined up to a global sign). If $\theta=\sum_{i=1}^{n} p_{i} d q_{i}, \theta$ vanishes on $F$ and $\omega=d \theta$. If $L, \nabla$ is a prequantization of $(X, \omega), L$ has a nowhere vanishing section $s_{0}$ with $\nabla_{\xi} s_{0}=2 \pi i \theta(\xi) s_{0}$. Also $d q_{1} \wedge \cdots \wedge d q_{n}$ is closed, so $\nabla_{\xi}^{1 / 2}\left(d q_{1}\right.$ $\left.\wedge \cdots \wedge d q_{n}\right)^{1 / 2}=0$ for all $\xi \in \Gamma F$. Thus $\Gamma_{F} L \otimes Q^{F}$ has elements of the form $\varphi s_{0} \otimes\left(d q_{1} \wedge \cdots \wedge d q_{n}\right)^{1 / 2}$ with $\partial \varphi / \partial p_{i}=0, i=1, \ldots, n$. Thus $\varphi$ is a function of $q_{1}, \ldots, q_{n}$ only. Then $s_{0}$ can be normalized so that $\left|s_{0}\right|^{2} \equiv 1$, and $\left\langle\left(d q_{1} \wedge \cdots \wedge d q_{n}\right)^{1 / 2}, \quad\left(d q_{1} \wedge \cdots \wedge d q_{n}\right)^{1 / 2}\right\rangle$ projects to the density $d q_{1} \cdots d q_{n}$ on $\mathbf{R}^{n}$. Thus

$$
\left\|\varphi s_{0} \otimes\left(d q_{1} \wedge \cdots \wedge d q_{n}\right)^{1 / 2}\right\|^{2}=\int_{\mathbf{R}^{n}}\left|\varphi\left(q_{1}, \ldots, q_{n}\right)\right|^{2} d q_{1} \cdots d q_{n} .
$$

In this case then, $\mathfrak{\mathcal { B }}_{F}=L^{2}\left(\mathbf{R}^{n}\right)$.

A second polarization $G$ arises from the identification $\mathbf{R}^{2 n}=\mathbf{C}^{n}$. Put $z_{j}=q_{j}+i p_{j}, j=1, \ldots, n$ and let $G$ be spanned by $\partial / \partial \bar{z}_{1}, \ldots, \partial / \partial \bar{z}_{n}$. Then $K^{G}$ is spanned by $d z_{1} \wedge \cdots \wedge d z_{n}$ and $Q^{G}$ by $\left(d z_{1} \wedge \cdots \wedge d z_{n}\right)^{1 / 2}$. Let $\theta^{\prime}=i / 2 \sum_{j=1}^{n} \bar{z}_{j} d z_{j}$ so that $\omega=d \theta^{\prime}$ and $\theta^{\prime}$ vanishes on $G$. We have a nowhere vanishing section $t_{0}$ of $L$ with $\nabla_{\xi} t_{0}=2 \pi i \theta^{\prime}(\xi) t_{0}$. Then $t_{0}=\varphi_{0} s_{0}$ for some nowhere vanishing function $\varphi_{0}$. According to [4], $\varphi_{0}$ is given by $d \log \varphi_{0}=$ $2 \pi i\left(\theta^{\prime}-\theta\right)$ which may be solved to give

$$
\varphi_{0}=\exp \left\{-\pi|z|^{2} / 2-i \pi \sum_{j=1}^{n} q_{j} p_{j}\right\} .
$$

Then $\left|t_{0}\right|^{2}=\left|\varphi_{0}\right|^{2}=\exp \left(-\pi|z|^{2}\right)$. Any element $t \in \Gamma_{G} L \otimes Q^{G}$ has the form $t=\psi t_{0} \otimes\left(d z_{1} \wedge \cdots \wedge d z_{n}\right)^{1 / 2}$ with $\psi$ holomorphic, and since

$$
\left(d z_{1} \wedge \cdots \wedge d z_{n}\right) \wedge \overline{\left(d z_{1} \wedge \cdots \wedge d z_{n}\right)}=(2 i)^{n} \lambda,
$$

we obtain $\left\langle\left(d z_{1} \wedge \cdots \wedge d z_{n}\right)^{1 / 2},\left(d z_{1} \wedge \cdots \wedge d z_{n}\right)^{1 / 2}\right\rangle=|\lambda|$ and so 


$$
\|t\|^{2}=\int_{\mathbf{R}^{2 n}}\left|\psi\left(z_{1}, \ldots, z_{n}\right)\right|^{2}\left(\exp \left(-\pi|z|^{2}\right)\right)|\lambda| .
$$

It follows that $\mathscr{B}_{G}$ may be identified with the holomorphic functions on $\mathbf{C}^{n}$ square integrable for the Gaussian measure $\left(\exp \left(-\pi|z|^{2}\right)\right)|\lambda|$.

These polarizations $F$ and $G$ on $\mathrm{R}^{2 n}$ are easily paired since $F \cap \bar{G}=0$ and

$$
\left(d q_{1} \wedge \cdots \wedge d q_{n}\right) \wedge \overline{\left(d z_{1} \wedge \cdots \wedge d z_{n}\right)}=(i)^{n} \lambda
$$

so that $\left\langle\left(d q_{1} \wedge \cdots \wedge d q_{n}\right)^{1 / 2},\left(d z_{1} \wedge \cdots \wedge d z_{n}\right)^{1 / 2}\right\rangle=1$. Hence

$$
\begin{aligned}
\left\langle\varphi s_{0} \otimes(\right. & \left.\left(d q_{1} \wedge \cdots \wedge d q_{n}\right)^{1 / 2}, \psi t_{0} \otimes\left(d z_{1} \wedge \cdots \wedge d z_{n}\right)^{1 / 2}\right\rangle \\
& =\int_{\mathbf{R}^{2 n}} \varphi\left(q_{1}, \ldots, q_{n}\right) \overline{\psi\left(z_{1}, \ldots, z_{n}\right)} \exp \left\{-\pi|z|^{2} / 2+i \pi p \cdot q\right\}|\lambda| .
\end{aligned}
$$

As a map from $\mathscr{B}_{G}$ to $\mathfrak{S}_{F}$ this is formally given by

$$
(T \psi)(q)=\int_{\mathbf{R}^{n}} \psi(q+i p) \exp \left\{-\pi\left(p^{2}+q^{2}\right) / 2-i \pi p \cdot q\right\} d^{n} p .
$$

If $\psi$ is a polynomial, it is in $\mathscr{B}_{G}$ and $T \psi \in \mathfrak{B}_{F}$. Since polynomials are dense in $\mathfrak{Q}_{G}, T$ is densely defined. Proving $T$ is unitary is messy using polynomials, so instead we use that $\mathfrak{B}_{G}$ has a reproducing kernel.

If $\psi_{w}(z)=\exp \pi \bar{w} \cdot z, \psi_{w} \in \mathscr{B}_{G}$ for all $w \in \mathbf{C}^{n},\left(\left\|\psi_{w}\right\|^{2}=\exp \pi|w|^{2}\right)$, and for any $\psi \in \mathfrak{B}_{G}, \psi(w)=\left(\psi, \psi_{w}\right)$.

Then finite linear combinations $\Sigma_{\alpha} c_{\alpha} \psi_{w_{\alpha}}$ are dense in $\mathfrak{B}_{G}$ also, so we need only compute $T \psi_{w}$. This is a Gaussian integral and can be computed explicitly:

$$
\left(T \psi_{w}\right)(q)=2^{n / 2} \exp \left\{-\pi q^{2}-\pi \bar{w}^{2} / 2+2 \pi \bar{w} \cdot q\right\} .
$$

Again $\left(T \psi_{w}, T \psi_{v}\right)$ is a Gaussian integral and may be evaluated as

$$
\left(T \psi_{w}, T \psi_{v}\right)=\exp \pi v \cdot \bar{w}=\left(\psi_{w}, \psi_{v}\right)
$$

Thus $T$ is an isometry on the dense domain above. If it has dense range it extends to a unitary map of $\mathfrak{G}_{G}$ onto $\mathfrak{S}_{F}$. That the range is dense follows because $\left(T \psi_{w}\right)(q)$ is essentially the generating function for the Hermite functions whose linear combinations are dense in $L^{2}\left(\mathbf{R}^{n}\right)$.

Using the reproducing kernel,

$$
\begin{aligned}
(T \psi)(q) & =\int_{\mathbf{R}^{n}}\left(\psi, \psi_{q+i p}\right) \exp \left\{-\pi\left(p^{2}+q^{2}\right) / 2-i \pi p \cdot q\right\} d^{n} p \\
& =\int_{\mathbf{R}^{2 n}} \psi(z) K(z, q)\left(\exp \left(-\pi|z|^{2}\right)\right)|\lambda|
\end{aligned}
$$

with $K(z, q)=\left(T \psi_{z}\right)(q)$. Apart from normalization, $K$ is Bargmann's transform [1] from $\mathscr{S}_{G}$ to $\mathscr{S}_{F}$. 
3. The real and complex polarizations of $T_{0}^{*} S^{n} . T_{0}^{*} S^{n}$ can be identified with $X=\left\{(e, x) \in \mathbf{R}^{n+1} \times \mathbf{R}^{n+1} \mid e \cdot e=1, x \cdot e=0, x \neq 0\right\}$. The natural symplectic structure on $T_{0}^{*} S^{n}$ carries over to $\omega$ on $X$ where $\omega=d \theta, \theta=x \cdot d e$, regarding the components $e_{0}, \ldots, e_{n}, x_{0}, \ldots, x_{n}$ as functions on $X$. For $n>3, X$ is simply-connected but $\pi_{1}(X)=Z_{2}$ if $n=2$. To avoid technical complications arising from nonsimple-connectedness we shall assume $n>3$.

$X$ fibres over $S^{n}=\left\{e \in \mathbf{R}^{n+1} \mid e \cdot e=1\right\}$ and the fibres are the cotangent spaces with the origin deleted. Put $\pi(e, x)=e$.

Let $|x|=(x \cdot x)^{1 / 2}, h(e, x)=2 \pi|x|$, then $h \in C(X)$ and $\xi_{h}$ generates a flow $\sigma_{t}$ which may be found to be

$$
\sigma_{t}(e, x)=((\cos 2 \pi t) e+(\sin 2 \pi t) x /|x|,(\cos 2 \pi t) x-(\sin 2 \pi t)|x| e) .
$$

This may be more neatly expressed by introducing $z \in \mathbf{C}^{n+1}$ with

$$
z=|x| e+i x
$$

and then $\sigma_{t} z=(\exp (-2 \pi i t)) z .(e, x) \mapsto z$ injects $X$ into $\mathbf{C}^{n+1}$ and the image is the nonsingular cone $\left\{z \in \mathbf{C}^{n+1} \mid z \cdot z=0, z \neq 0\right\}$, giving $X$ a complex structure. Let $d=\partial+\bar{\partial}$ be the usual decomposition of the exterior derivative into components of type $(1,0)$ and $(0,1)$.

Of course $\bar{\partial} z_{i}=0, i=0, \ldots, n$. From (1) $z \cdot \bar{z}=2|x|^{2}$ so $4|x| \partial|x|=2 \partial|x|^{2}$ $=\bar{z} \cdot d z=2|x| d|x|-2 i|x| x \cdot d e$. Thus $\theta=i \partial|x|-i \bar{\partial}|x|$ and hence

$$
\omega=2 i \bar{\partial} \partial|x| \text {. }
$$

This shows that $\omega$ is the Kaehler 2-form of a positive definite Hermitian metric and hence that the tangents of type $(0,1)$ form a positive polarization $G$ with $G \cap \bar{G}=0$. Let $F=\operatorname{Ker} \pi_{*}$ be the tangent spaces to the fibering $\pi$ : $X \rightarrow S^{n}$. Since $\sigma_{t^{*}} G=G, h \in C_{G}^{1}$. However, $h \notin C_{F}^{1}$ (though $h^{2} \in C_{F}^{2}$ ).

Let $L, \nabla$ be a prequantization of $(X, \omega)$. Then $\omega=d \theta$ implies the existence of a nowhere vanishing section $s_{F}$ with $\nabla_{\xi} s_{F}=2 \pi i \theta(\xi) s_{F} . \theta$ is real so $\left|s_{F}\right|^{2}$ is constant and $s_{F}$ can be normalized so $\left|s_{F}\right|^{2}=1$.

Similarly $\omega=d(2 i \partial|x|)$ so we have $s_{G}$ with $\nabla_{\xi} s_{G}=-4 \pi \partial|x|(\xi) s_{G}$. But $s_{G}=\varphi_{0} s_{F}$ for some nowhere vanishing function $\varphi_{0}$ and

$$
d \log \varphi_{0}=2 \pi i(2 i \partial|x|-\theta)=-2 \pi d|x|
$$

so $\varphi_{0}=\exp (-2 \pi|x|)$ apart from a constant which we can set equal to 1 . Thus $\left|s_{G}\right|^{2}=\left|\varphi_{0}\right|^{2}=\exp (-4 \pi|x|)$. This completes the analysis of the prequantization.

To discover whether half-forms exist, consider $K^{F}$. Let $\rho$ be any $n$-form on $S^{n}$ then $\pi^{*} \rho$ is an $n$-form vanishing on $F$ so $\pi^{*} \rho \in K^{F}$. Since $S^{n}$ is orientable we can choose $\rho$ nowhere vanishing, and then $\pi^{*} \rho$ vanishes nowhere, showing $K^{F}$ is trivial. Thus there is a square root $Q^{F}$, unique since $X$ is simply-connected. The same conclusion could have been reached from [5] since it is known that when $F$ is the tangent bundle to a projection $\pi: X \rightarrow Y$ the $\bmod 2$ 
reduction of the Chern class of $F$ is the square of the first Stiefel-Whitney class of $Y$, pulled back to $X$. Then, if $Y$ is orientable, the Chern class must be even.

Observe also that since $\rho$ is a form of maximum degree on $S^{n}, d \rho=0$ so that $d \pi^{*} \rho=0$ and hence $\nabla_{\xi} \pi^{*} \rho=0, \xi \in \Gamma F$. Fix $\rho_{0}$ as the Riemannian volume on $S^{n}$, which in terms of the functions $e_{i}$ is

$$
\rho_{0}=\sum_{j=0}^{n}(-1)^{j} e_{j} d e_{0} \wedge \cdots \wedge \widehat{d e}_{j} \wedge \cdots \wedge d e_{n}
$$

where $\widehat{d e_{j}}$ means that term is omitted. On the set where $e_{k} \neq 0$ we can take $e_{0}, \ldots, e_{k-1}, e_{k+1}, \ldots, e_{n}$ as coordinates and obtain

$$
\rho_{0}=(-1)^{k} e_{k}^{-1} d e_{0} \wedge \cdots \wedge \widehat{d e}_{k} \wedge \cdots \wedge d e_{n} .
$$

Expression (3) makes sense on $X$ and gives $\pi^{*} \rho_{0}$.

Let $Q^{F} \otimes Q^{F}=K^{F}$ and $\mu_{F}$ be a section of $Q^{F}$ with $\mu_{F} \otimes \mu_{F}=\pi^{*} \rho_{0}$, which exists since $X$ is simply-connected. Then also $\nabla_{\xi}^{1 / 2} \mu_{F}=0$ for all $\xi$ in $\Gamma F$.

$K^{G}$ may be handled similarly. We look for a section $\sigma$ which has an expression analogous to (3) in terms of the functions $z_{i}$ instead of $e_{i}$, and in order that $d \sigma=0$ one finds

$$
\sigma=|x|^{-2} \sum_{j=0}^{n}(-1)^{j} \bar{z}_{j} d z_{0} \wedge \cdots \wedge \widehat{d z_{j}} \wedge \cdots \wedge d z_{n} .
$$

If $U_{j} \subset X$ is the subset where $e_{j} \neq 0$, then $z_{j} \neq 0$ on $U_{j}$ and

$$
\sigma \mid U_{j}=2(-1)^{j} z_{j}^{-1} d z_{0} \wedge \cdots \wedge \widehat{d z}_{j} \wedge \cdots \wedge d z_{n} .
$$

Thus $\sigma$ vanishes nowhere and $\nabla_{\xi} \sigma=0, \xi \in \Gamma G$. Let $Q^{G} \otimes Q^{G}=K^{G}$ and $\mu_{G}$ be the section of $Q^{G}$ with $\mu_{G} \otimes \mu_{G}=\sigma$, so that $\nabla_{\xi}^{1 / 2} \mu_{G}=0$ for $\xi$ in $\Gamma G$.

We have thus shown $\Gamma_{F} L \otimes Q^{F}$ consists of sections of the form $\varphi \circ \pi s_{F} \otimes$ $\mu_{F}$ with $\varphi \in C^{\infty}\left(S^{n}\right)$, and $\Gamma_{G} L \otimes Q^{G}$ of the form $\psi s_{G} \otimes \mu_{G}$ with $\psi$ holomorphic. The norms are easily computed as in $\S 2$. $F$ is real so $\left\langle\mu_{F}, \mu_{F}\right\rangle=\pi^{*}\left|\rho_{0}\right|$, and $\|\varphi\|_{F}^{2}=\int_{S^{n}}|\varphi|^{2}\left|\rho_{0}\right|$, so $\mathfrak{Q}_{F}$, the completion of $\Gamma_{F} L \otimes Q^{F}$ coincides with $L^{2}\left(S^{n},\left|\rho_{0}\right|\right)$.

For $G$ we have $G \cap \bar{G}=0$, so $i^{n}\langle\sigma, \sigma\rangle \lambda=\sigma \wedge \bar{\sigma}$ gives $\langle\sigma, \sigma\rangle=$ $2^{n+2}|x|^{n-2}$ and so $\left\langle\mu_{G}, \mu_{G}\right\rangle=2^{n / 2+1}|x|^{n / 2-1}$. Thus

$$
\|\psi\|_{G}^{2}=\int_{X}|\psi|^{2}(\exp (-4 \pi|x|)) 2^{n / 2+1}|x|^{n / 2-1}|\lambda| .
$$

$\mathfrak{S}_{G}$ is then all holomorphic functions $\psi$ on $X$ with $\|\psi\|_{G}$ finite. The exponential convergence factor means $\mathfrak{G}_{G}$ contains all polynomials in $z_{0}, \ldots, z_{n}$ so is not trivial.

To pair $F$ and $G$ we need to compute $\pi^{*} \rho_{0} \wedge \bar{\sigma}$. This is easily done on $U_{j}$ using formulas (4) and (5) and the following expression 


$$
\begin{aligned}
\lambda \mid U_{j}= & 2 e_{j}^{-2} d e_{0} \wedge \cdots \wedge \widehat{d e_{j}} \wedge \cdots \wedge d e_{n} \\
& \wedge d x_{0} \wedge \cdots \wedge \widehat{d x_{j}} \wedge \cdots \wedge d x_{n} .
\end{aligned}
$$

One finds $\pi^{*} \rho_{0} \wedge \bar{\sigma}=2(-1)^{n}|x|^{-1} \lambda$. Thus $\left\langle\mu_{F}, \mu_{G}\right\rangle=i^{n} 2^{1 / 2}|x|^{-1 / 2}$. We shall drop the factor $i^{n}$ since it makes no difference to the existence or unitarity. Denote the pairing of $\varphi s_{F} \otimes \mu_{F}$ and $\psi s_{G} \otimes \mu_{G}$ by $\langle\varphi, \psi\rangle$, then

$$
\langle\varphi, \psi\rangle=\int_{X} \varphi \bar{\psi}(\exp (-2 \pi|x|)) 2^{1 / 2}|x|^{-1 / 2}|\lambda| .
$$

As a formal map $T: \mathfrak{B}_{G} \rightarrow \mathfrak{B}_{F}$ the pairing can be written

$$
(T \psi)(e)=2^{1 / 2} \int_{x \cdot e=0} \psi(|x| e+i x)(\exp (-2 \pi|x|))|x|^{-1 / 2} d^{n} x
$$

with $d^{n} x$ the normalized Lebesgue measure on the cotangent space $\pi^{-1}(e)$.

4. Existence and nonunitary nature of the pairing. The proof of the existence of the pairing is based on being able to write down a kernel $K(z, e)$ analogous to that of $\$ 2$. If $x \in \mathbf{R}^{n+1}, z \in \mathbf{C}^{n+1}$ and $\Delta_{x}$ denotes the Laplacian in the $x$-variables then

$$
\Delta_{x}(x \cdot z)^{k}=k(k-1) z \cdot z(x \cdot z)^{k-2},
$$

from which it follows that if $z \in X,(x \cdot z)^{k}$ is a homogeneous harmonic polynomial and therefore its restriction to the unit sphere is a spherical harmonic. For $x$ fixed, as a function of $z,(x \cdot z)^{k}$ is holomorphic and polynomial and thus in $\mathfrak{S}_{G}$. The spherical harmonics are dense in $\mathfrak{B}_{F}$ and it will be shown that the polynomials in $z$ are dense in $\mathfrak{B}_{G}$. These will provide dense domains for $T$ and $T^{-1}$.

Let $\mathcal{S}_{k}$ denote the spherical harmonics of order $k$ on $S^{n}$ and $\mathscr{P}_{k}$ the polynomials homogeneous of degree $k$ on $X$. Then

$$
\operatorname{dim} \mathcal{S}_{k}=\operatorname{dim} \mathscr{P}_{k}=(2 k+n-1) \Gamma(k+n-1) /\{\Gamma(n) \Gamma(k+1)\}
$$

(this equality of dimension could be derived from our analysis of the relationship between $\delta_{k}$ and $\mathscr{P}_{k}$ by working a little harder). Our first objective is to show $T$ maps $\mathscr{P}_{k}$ isomorphically onto $\mathscr{S}_{k}$ : this is the Laplace representation of elements of $\mathfrak{S}_{k}$.

Define, for $\varphi \in \mathcal{S}_{k}, A_{k} \varphi \in \mathscr{P}_{k}$ by

$$
\left(A_{k} \varphi\right)(z)=\int_{S^{n}} \varphi(a)(a \cdot z)^{k}\left|\rho_{0}\right|(d a),
$$

and for $\psi \in \mathscr{P}_{k}$ define $B_{k} \psi \in C\left(S^{n}\right)$ by

$$
\left(B_{k} \psi\right)(a)=2^{n / 2+1} \int_{X}(a \cdot \bar{z})^{k} \psi(z)(\exp (-4 \pi|x|))|x|^{n / 2-1}|\lambda| .
$$

The exponential convergence of the integrand justifies all the following manipulation. 


$$
\begin{aligned}
& \left(B_{k} \circ A_{k} \varphi\right)(a) \\
& \quad=2^{n / 2+1} \int_{X}(a \cdot \bar{z})^{k} \int_{S^{n}} \varphi(b)(b \cdot z)^{k}\left|\rho_{0}\right|(d b)(\exp (-4 \pi|x|))|x|^{n / 2-1}|\lambda| \\
& \quad=\int_{S^{n}} \varphi(b) F(a, b)\left|\rho_{0}\right|(d b),
\end{aligned}
$$

where $F(a, b)=2^{n / 2+1} \int_{X}(a \cdot \bar{z})^{k}(b \cdot z)^{k}(\exp (-4 \pi|x|))|x|^{n / 2-1}|\lambda| . F(a, b)$ is a kernel defining a map of $\delta_{k}$ to itself and clearly is $O(n+1)$ invariant. But $\delta_{k}$ is an irreducible representation of $O(n+1)$, so $B_{k} \circ A_{k}$ must be a multiple $a_{k}$ of the identity. To find $a_{k}$ we set $a=b$ and integrate

$$
a_{k} \operatorname{dim} \delta_{k} \operatorname{vol} S^{n}=2^{n / 2+1} \int_{S^{n}} F(a, a)\left|\rho_{0}\right|(d a)
$$

But $O(n+1)$ is transitive on $S^{n}$ so $F(a, a)$ is constant. We can set $a=\varepsilon_{n+1}$, the $(n+1)$ th coordinate direction in $\mathbf{R}^{n+1}$ and then

$$
a_{k} \operatorname{dim} \delta_{k}=2^{n / 2+1} \int_{X}\left|z_{n+1}\right|^{2 k}(\exp (-4 \pi|x|))|x|^{n / 2-1}|\lambda|
$$

This integral is evaluated in the appendix to give

$$
\begin{aligned}
a_{k}= & 2^{-4 k-3 n+5} \pi^{2 k-n / 2+3 / 2} \\
& \cdot \frac{\Gamma(2 k+3 n / 2-1) \Gamma(n) \Gamma(k+1)^{2}}{(2 k+n-1) \Gamma(n / 2) \Gamma(k+(n+1) / 2) \Gamma(k+n-1)} .
\end{aligned}
$$

This is nonzero so $A_{k}$ and $B_{k}$ are invertible.

$\mathfrak{B}_{G}$ is a unitary representation of $O(n+1)$ and by the above, $O(n+1)$ acts irreducibly on $\mathscr{P}_{k}$, so $\bigoplus_{k=0}^{\infty} \mathscr{P}_{k}$ is an orthogonal direct sum within $\mathfrak{B}_{G}$. But in Lemma 1 of [9] I showed a holomorphic function $f$ on $X$ had an expansion $f=\sum_{k=0}^{\infty} f_{k}$ with $f_{k} \in \mathscr{P}_{k}$ so that $\mathscr{Q}_{G}=\bigoplus_{k=0}^{\infty} \mathscr{P}_{k}$. Let $\mathscr{P}=\amalg_{k=0}^{\infty} \mathscr{P}_{k}$ be the algebraic sum. This is thus a dense domain in $\mathscr{S}_{G}$.

Let $\psi_{1}, \psi_{2} \in \mathscr{P}_{k}$ then $A_{k}: \mathcal{S}_{k} \rightarrow \mathscr{P}_{k}$ is onto so $\psi_{i}=A_{k} \varphi_{i}$ with $\varphi_{i} \in \mathcal{S}_{k}$, $i=1,2$. Then

$$
\begin{aligned}
\left(\psi_{1}, \psi_{2}\right)_{G} & =\int_{X}\left(A_{k} \varphi_{1}\right)(z) \overline{\left(A_{k} \varphi_{2}\right)(z)}(\exp (-4 \pi|x|)) 2^{n / 2+1}|x|^{n / 2-1}|\lambda| \\
& =\int_{S^{n}} \int_{S^{n}} \varphi_{1}(a) \overline{\varphi_{2}(b)} F(a, b)\left|\rho_{0}\right|(d a)\left|\rho_{0}\right|(d b)
\end{aligned}
$$

by a simple rearrangement. $\operatorname{Thus}\left(\psi_{1}, \psi_{2}\right)_{G}=a_{k}\left(\varphi_{1}, \varphi_{2}\right)_{F}$. Hence $a_{k}^{-1 / 2} A_{k}$ is unitary. 
Now consider $T_{k}=T \mid \mathscr{P}_{k}$, and

$$
\begin{aligned}
\left(T_{k} \circ A_{k} \varphi\right)(e) & =2^{1 / 2} \int_{x \cdot e=0}\left(A_{k} \varphi\right)(|x| e+i x)(\exp (-2 \pi|x|))|x|^{-1 / 2} d^{n} x \\
& =\int_{S^{n}} \varphi(a) G(a, e)\left|\rho_{0}\right|(d a)
\end{aligned}
$$

with

$$
G(a, b)=2^{1 / 2} \int_{x \cdot b=0}\{a \cdot(|x| b+i x)\}^{k}(\exp (-2 \pi|x|))|x|^{-1 / 2} d^{n} x
$$

Again, $G(a, b)$ is $O(n+1)$-invariant and hence a multiple, $b_{k}$, of the identity. $b_{k}$ is found, as before, by setting $a=b$ and integrating:

$$
b_{k}=2^{-k-n+2} \pi^{-k-n / 2+1 / 2} \frac{\Gamma(k+n-1 / 2) \Gamma(n) \Gamma(k+1)}{(2 k+n-1) \Gamma(n / 2) \Gamma(k+n-1)} .
$$

Thus $T_{k}=b_{k} a_{k}^{-1} B_{k}$ and is $b_{k} a_{k}^{-1 / 2}$ times a unitary operator from $\mathscr{P}_{k}$ to $\mathcal{S}_{k}$. Also $\|T\|=\sup _{k} b_{k} a_{k}^{-1 / 2},\left\|T^{-1}\right\|=\sup _{k} b_{k}^{-1} a_{k}^{1 / 2}$, if these exist.

We calculate $b_{k}^{2} a_{k}^{-1}$ as

$$
\frac{2^{n / 2} \Gamma(k+n-1 / 2)^{2} \Gamma(k+(n-1) / 2)}{\operatorname{vol~} S^{n} \Gamma(k+n-1) \Gamma(k+3 n / 4) \Gamma(k+3 n / 4-1 / 2)} .
$$

This is monotone decreasing so $\|T\|=b_{0} a_{0}^{-1 / 2}$ is finite, and $\left\|T^{-1}\right\|=$ $\lim _{k \rightarrow \infty} b_{k}^{-1} a_{k}^{1 / 2}$. But

$$
\frac{\Gamma\left(k+\alpha_{1}\right) \cdots \Gamma\left(k+\alpha_{r}\right)}{\Gamma\left(k+\beta_{1}\right) \cdots \Gamma\left(k+\beta_{r}\right)}
$$

has the limit $\infty, 1$ or 0 as $k \rightarrow \infty$ according as $\sum_{i=1}^{r} \alpha_{i}$ is greater, equal to or less than $\Sigma_{i=1}^{r} \beta_{i}$. In our case $\alpha_{1}=\alpha_{2}=n-1 / 2, \alpha_{3}=(n-1) / 2, \beta_{1}=n-$ $1, \beta_{2}=3 n / 4, \beta_{3}=3 n / 4-1 / 2$ so $\alpha_{1}+\alpha_{2}+\alpha_{3}=5 n / 2-3 / 2=\beta_{1}+\beta_{2}$ $+\beta_{3}$, so that $\left\|T^{-1}\right\|=\left(\operatorname{vol} S^{n}\right)^{1 / 2} 2^{-n / 4}$, which is finite. Thus $T$ and $T^{-1}$ are bounded and hence we have established the rigorous existence of the pairing. Since $\left\|T_{k}\right\|$ is properly decreasing, $T$ is not unitary, nor a multiple of a unitary operator.

The flow $\sigma_{t}$ preserves $G$, so lifts into $L$ and satisfies $\sigma_{t} \cdot s_{G}=s_{G}$. Also one finds from (5) that

$$
\sigma_{i}^{*} \sigma=\exp \{-(n-1) 2 \pi i t\} \sigma
$$

and so $\sigma_{t}^{*} \mu_{G}=\exp \{-(n-1) \pi i t\} \mu_{G}$. Thus $\sigma_{t}$ quantizes on $\mathfrak{B}_{G}$ to give the unitary group $U_{t}$ with

$$
\left(U_{t} \psi\right)(z)=\exp (n-1) \pi i t \psi(\exp 2 \pi i t z) .
$$

For $\psi \in \mathscr{P}_{k}$ we have $U_{t} \psi=\exp \{(k+(n-1) / 2) 2 \pi i t\} \psi$, so that

$$
T U_{t} T^{-1}=\exp \left\{2 \pi i t\left[-\Delta+(n-1)^{2} / 4\right]^{1 / 2}\right\},
$$

as the latter group has the same spectrum and eigenspaces. 
Appendix. To evaluate

$$
C_{k}=\int_{X}\left|z_{n+1}\right|^{2}(\exp (-4 \pi|x|))|x|^{n / 2-1}|\lambda|,
$$

write $x=r y$ with $y \cdot y=1, y \cdot e=0$ then

$$
\begin{aligned}
C_{k} & =\int_{0}^{\infty} r^{2 k+3 n / 2} \exp (-4 \pi r) d r \int_{\substack{y \cdot e=0 \\
y \cdot y=e \cdot e=1}}\left(e_{n+1}^{2}+y_{n+1}^{2}\right)^{k} d \text { vol, } \\
& =(4 \pi)^{-2 k-3 n / 2+1} \Gamma(2 k+3 n / 2-1) I_{k}
\end{aligned}
$$

where

$$
I_{k}=\int_{\substack{y \cdot e=0 \\ y \cdot y=e \cdot e=1}}\left(a \cdot e^{2}+a \cdot y^{2}\right)^{k} d \text { vol. }
$$

This is independent of $a$, so integrating over $a$ gives

$$
\begin{aligned}
I_{k} \operatorname{vol} S^{n} & =\int_{S^{n}} \int_{\begin{array}{c}
y \cdot e=0 \\
e \cdot e=y \cdot y=1
\end{array}}\left(a \cdot e^{2}+a \cdot y^{2}\right)^{k} d \text { vol }\left|\rho_{0}\right|(d a) \\
& =\int_{\substack{y \cdot e=0 \\
e \cdot e=y \cdot y=1}} \int_{S^{n}}\left(a \cdot e^{2}+a \cdot y^{2}\right)^{k}\left|\rho_{0}\right|(d a) d \text { vol. }
\end{aligned}
$$

But $O(n+1)$ is transitive on the set of pairs $(e, y), e \cdot e=y \cdot y=1$, $e \cdot y=0$, so

$$
\int_{S^{n}}\left(a \cdot e^{2}+a \cdot y^{2}\right)^{k}\left|\rho_{0}\right|(d \alpha)
$$

is independent of $(e, y)$. We can therefore evaluate it by setting $e=\varepsilon_{n+1}$, $y=\varepsilon_{n}$. Then

$$
I_{k} \operatorname{vol} S^{n}=\operatorname{vol} S^{n} \operatorname{vol} S^{n-1} \int_{S^{n}}\left(e_{n+1}^{2}+e_{n}^{2}\right)^{k}\left|\rho_{0}\right|(d a)
$$

This last integral we write in spherical polar coordinates:

$$
\begin{aligned}
I_{k} & =\operatorname{vol} S^{n-1} \operatorname{vol} S^{n-2} \int_{0}^{\pi} \int_{0}^{\pi}\left(\cos ^{2} \theta+\sin ^{2} \theta \cos ^{2} \varphi\right)^{k} \sin ^{n-1} \theta \sin ^{n-2} \varphi d \theta d \varphi \\
& =\operatorname{vol} S^{n-1} \operatorname{vol} S^{n-2} \int_{0}^{\pi} \int_{0}^{\pi}\left(1-\sin ^{2} \theta \sin ^{2} \varphi\right)^{k} \sin ^{n-1} \theta \sin ^{n-2} \varphi d \theta d \varphi \\
& =\operatorname{vol} S^{n-1} \operatorname{vol} S^{n-2} \sum_{r=0}^{k}(-1)^{r}\left(\begin{array}{l}
k \\
r
\end{array}\right) J_{2 r+n-1} J_{2 r+n-2}
\end{aligned}
$$


where $J_{k}=\int_{0}^{\pi} \sin ^{k} \theta d \theta$. Now, integrating by parts,

$$
\begin{aligned}
J_{k} & =-\left.\sin ^{k-1} \theta \cos \theta\right|_{0} ^{\pi}+(k-1) \int_{0}^{\pi} \sin ^{k-2} \theta \cos ^{2} \theta d \theta \\
& =(k-1)\left(J_{k-2}-J_{k}\right)
\end{aligned}
$$

for $k \geqslant 2$. Thus $k J_{k}=(k-1) J_{k-2}$ for $k \geqslant 2$. Multiplying both sides by $J_{k-1}$, we see $k J_{k} J_{k-1}$ is constant, so $k J_{k} J_{k-1}=J_{1} J_{0}=2 \pi$. Thus

$$
\begin{aligned}
I_{k} & =\operatorname{vol} S^{n-1} \operatorname{vol} S^{n-2} \sum_{r=0}^{k}(-1)^{r}\left(\begin{array}{l}
k \\
r
\end{array}\right) \frac{2 \pi}{2 r+n-1} \\
& =2 \pi \operatorname{vol} S^{n-1} \operatorname{vol} S^{n-2} \int_{0}^{1} x^{n-2}\left(1-x^{2}\right)^{k} d x
\end{aligned}
$$

Another integration by parts procedure shows that the last integral is

$$
\frac{1}{2} \Gamma(k+1) \Gamma((n-1) / 2) / \Gamma(k+(n+1) / 2) .
$$

Then $I_{k}=4 \pi^{n+1 / 2} \Gamma(k+1) /\{\Gamma(n / 2) \Gamma(k+(n+1) / 2)\}$.

\section{REFERENCES}

1. V. Bargmann, On a Hilbert space of analytic functions and an associated integral transform, Comm. Pure Appl. Math. 20 (1967), 1-101.

2. R. Blattner, The metalinear geometry of nonreal polarizations, Lecture Notes in Math., vol. 570, Springer-Verlag, Berlin and New York, 1977.

3. J. Elhadad, Quantification du flot géodésique de la sphère $S^{n}$, Centre National de la Recherche Scientifique, Marseille, June 1977.

4. B. Kostant, Quantization and unitary representations. I, Lecture Notes in Math., vol. 170, Springer-Verlag, Berlin and New York, 1970. 1974.

5. B. Kostant, Symplectic spinors, Symposia Mathematica XIV, Academic Press, London,

6. , On the definition of quantization, Colloque symplectique (Aix-en-Provence), Centre National de la Recherche Scientifique, 1974.

7. J. Moser, Regularization of Kepler's problem and the averaging method on a manifold, Comm. Pure Appl. Math. 23 (1970), 609-636.

8. C. Müller, Spherical harmonics, Lecture Notes in Math., vol. 17, Springer-Verlag, Berlin and New York, 1966.

9. L. Nirenberg, A complex Frobenius theorem, Seminars on analytic functions. I, Princeton Univ. Press, Princeton, N.J., 1957, pp. 172-189.

10. J. Rawnsley, Coherent states and Kaehler manifolds, Quart. J. Math. Oxford Ser. 28 (1977), 403-415.

11. On the pairing of polarizations, Comm. Math. Phys. 58 (1978), 1-8.

12. On the cohomology groups of a polarization and diagonal quantization, Trans. Amer. Math. Soc. 230 (1977), 235-255.

13. D. Simms and N. Woodhouse, Lectures on geometric quantization, Lecture Notes in Physics, vol. 53, Springer-Verlag, Berlin and New York, 1976. 
14. J.-M. Souriau, Structure des systèmes dynamiques, Dunod, Paris, 1970.

15. ___ Sur la variété de Kepler, Symposia Mathematica XIV, Academic Press, London, 1974.

16. A. Weinstein, Quasi-classical mechanics on spheres, Symposia Mathematica XIV, Academic Press, London, 1974.

School of Thioretical Physics, Dublin Institute for Advanced Studies, Dublin 4, IRELAND

Current address: Department of Mathematics, University of California, Berkeley, California 94720 